\title{
Anti-colonic Inflammation by Black Raspberries through Regulating Toll-like Receptor-4 Signaling in Interlukin-10 Knockout Mice
}

\author{
Yi-Wen Huang ${ }^{1}$, Carla Elena Echeveste ${ }^{2}$, Kiyoko Oshima ${ }^{3}$, Jianying Zhang ${ }^{4}$, Martha Yearsley ${ }^{5}$, \\ Jianhua $\mathrm{Yu}^{6, \star}$, Li-Shu Wang ${ }^{2, *}$ \\ ${ }^{1}$ Department of Obstetrics and Gynecology, ${ }^{2}$ Division of Hematology and Oncology, Department of Medicine, Medical \\ College of Wisconsin, Milwaukee, WI, ${ }^{3}$ Department of Pathology, Johns Hopkins University School of Medicine, Baltimore, \\ MD, ${ }^{4}$ Division of Biostatistics, Department of Science of Informatics, City of Hope National Medical Center and Beckman \\ Research Institute, Duarte, CA, ${ }^{5}$ Department of Pathology, The Ohio State University Wexner Medical Center, Columbus, \\ $\mathrm{OH},{ }^{6}$ Department of Hematology and Hematopoietic Cell Transplantation, City of Hope National Medical Center and \\ Beckman Research Institute, Duarte, CA, USA
}

\begin{abstract}
Ulcerative colitis (UC) is a chronic inflammatory disease of the colon, with a steadily rising prevalence in Western and newly industrialized countries. UC patients have a cancer incidence as high as 10\% after 20 years of the disease. Although the importance of fruits and vegetables in defense against UC is beginning to be appreciated, the mechanisms remain largely unclear. In the current study, we reported that dietary black raspberries (BRBs) decreased colonic inflammation in the mucosa and submucosa of interleukin (IL)-10 knockout (KO) mice. We then used colon, spleen, and plasma from those mice to investigate whether BRBs exert their anti-inflammatory effects by correcting dysregulated toll-like receptor (TLR)-4 signaling to downregulate prostaglandin E2 (PGE2). Other studies reported that spleen is the reservoir of macrophages and depletion of macrophages in IL-10 KO mice prevents the development of colitis. Our results showed that BRBs decreased the percentages of macrophages in spleens of IL-10 KO mice. Moreover, mechanistically, the BRB diet corrected dysregulated TLR-4 signaling in cells from the colon and spleen, decreased PGE2 and prostaglandin I2, and increased 15-lipoxygenase and its product, 13-S-hydroxyoctadecadienoic acid, in plasma of IL$10 \mathrm{KO}$ mice. Therefore, we have elucidated one of the anti-inflammatory mechanisms of BRBs, and have identified biomarkers that could be indicators of response in UC patients treated with them. Our findings with BRBs could well apply to many other commonly consumed fruits and vegetables.
\end{abstract}

Key Words Ulcerative colitis, Black raspberries, Toll-like receptor-4 pathway, Spleen, interleukin-10 knockout mice

\section{INTRODUCTION}

Ulcerative colitis (UC) is a chronic inflammatory disease of the colon, and its prevalence is steadily rising in Western and newly industrialized countries [1]. UC patients have a cancer incidence as high as $10 \%$ after 20 years of the disease, so patients with longstanding colitis must be monitored for cancer development. Therefore, preventing the progression of UC to cancer remains an important goal, and chemoprevention is a promising approach. The mainstay for treating UC is 5-aminosalicylic acid (5-ASA), an anti-inflammatory medication [2] with few side effects. Individuals refractory to 5-ASA treatment require more aggressive treatment, including steroids and anti-TNF drugs. These medications are effective, but they carry significant long-term side effects and potential toxicities. There is a clear need for additional UC therapy that would be relatively nontoxic and complementary to 5-ASA medication.

Black raspberries (BRBs) are a natural food rich in protective anti-oxidant and anti-inflammatory compounds such

Received May 17, 2020, Revised June 2, 2020, Accepted June 9, 2020

Correspondence to Li-Shu Wang, E-mail: liswang@mcw.edu, https://orcid.org/0000-0002-6500-6943

Check for updates

Jianhua Yu, E-mail: jiayu@coh.org, https://orcid.org/0000-0002-0326-3223

*These authors contributed equally to this work as co-correspondence authors.

(i) (\$) This is an Open Access article distributed under the terms of the Creative Commons Attribution Non-Commercial License, which permits unrestricted noncommercial use, distribution, and reproduction in any medium, provided the original work is properly cited.

Copyright $\odot 2020$ Korean Society of Cancer Prevention 
as anthocyanins. We have shown that dietary BRBs reduce chronic inflammation by regulating methylation of Wnt pathway genes in interleukin (IL)-10 knockout (KO) mice, a model of UC [3]. In another mouse model that induces UC with dextran sodium sulfate, a BRB diet reduced colonic injury $[4,5]$. This protective effect was associated with regulation of methylation of Wnt pathway genes [4] and reduced levels of inflammatory cytokines, such as TNF- $\alpha$, IL-1 $\beta$, COX-2, prostaglandin E2 (PGE2), etc. in colon tissues [5]. BRB studies in humans also suggest that BRBs protect against colorectal cancer [6,7] and familial adenomatous polyposis [8]. In both studies, berries were well tolerated and had minimal side effects. Because of these advantages and BRBs' protective effects in the colon, it is highly likely that the berries will benefit patients with UC.

IL-10 affects the growth and differentiation of many hemopoietic cells in vitro; in particular, it is a potent suppressor of macrophage inflammatory functions [9]. Conventionally bred IL-10 KO mice spontaneously develop colitis because their macrophages preferentially differentiate into pro-inflammatory subsets that produce large amounts of IL-12, IL-23, and TNF- $\alpha$ [10]. Although circulating monocytes migrate to the colonic mucosa and rapidly respond to microbial triggers in the lumen, depletion of macrophages prevents IL-10 KO mice from developing colitis $[10,11]$. In contrast, IL-10 KO mice kept under specific pathogen-free conditions develop only local inflammation, which is limited to the proximal colon [9]. These results indicate that the bowel inflammation in IL$10 \mathrm{KO}$ mice originates from uncontrolled immune responses stimulated by microbial antigens and that IL-10 is an essential immune regulator in the intestinal tract [9]. They also indicate that macrophages are one of the key participants in colonic immune defenses $[9,11]$. Although IL-10 effectively treats colitis in animals, recombinant IL-10 induced only modest responses in patients with mild-to-moderate UC [12]. One possible reason is failure to achieve high local concentrations of IL-10 in the colon [13].

Toll-like receptors (TLRs) are broadly distributed in immune cells and intestinal epithelial cells [14]. They are the immune sensors of invading pathogens and microbial products. They activate signaling pathways that induce the expression of immune and pro-inflammatory genes. However, TLRs have also been implicated in several immune-mediated and inflammatory diseases, including UC [14]. There are 13 TLRs, TLR-1 to TLR-13, and except for TLR-4, most signaling helps maintain intestinal homeostasis [14]. In contrast, TLR-4 functions through its adaptor, MYD-88, to activate NF- $\mathrm{KB}$ which, in turn, upregulates COX-2 and PGE2 expression and promotes cell proliferation [14,15]. However, TLR-4-mediated inflammatory signaling is inhibited by anti-inflammatory agents such as prostaglandin 15-deoxy- $\Delta^{12,14}$-prostaglandin $\mathrm{J}_{2}$ [16]. In addi-

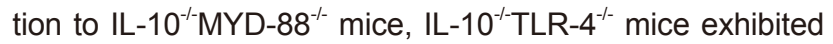
less colitis than IL-10 ${ }^{-/}$mice, indicating that TLR-4 signaling plays an important role in inducing colitis [17]. Through as- yet-unknown mechanisms, TLR-4 expression is upregulated in patients with UC [14]. Chronic inflammation probably increases the expression of TLR-4 in colonic epithelial cells, although, in humans, it is unclear whether this is a cause or an effect of pro-inflammatory cytokine secretion [14].

In the current study, we showed that BRBs decreased the percentages of macrophages in spleens of IL-10 wild type (WT) mice, which are the reservoir of macrophages [18]. Mechanistically, BRBs significantly decreased serum amyloid A (SAA), an apo-lipoprotein produced by the liver responding to proinflammatory cytokines from activated monocytes, in the circulating blood of IL-10 KO mice. Furthermore, the BRB diet decreased upregulated TLR-4 signaling in cells from the colon and spleen, decreased PGE2 and prostaglandin I2 (PGI2) (eicosanoids in the COX pathway), and increased 15-lipoxygenase (15-LOX) and its product, 13-S-hydroxyoctadecadienoic acid (13-S-HODE) (eicosanoids in the lipoxygenase pathway), in plasma of IL-10 KO mice. Therefore, our results elucidated the anti-inflammatory mechanisms of BRBs and identified pathways and/or biomarkers that could be indicators of response in UC patients treated with those berries.

\section{MATERIALS AND METHODS}

\section{Animals and BRB treatment}

All protocols were carried out in accordance with the institutional guidelines for animal care dictated by the Medical College of Wisconsin Animal Care and Use Committee (AUA2430). Animals and BRB treatments were described in our previous publication [3]. Briefly, 3-4-week-old WT and IL-10 KO male mice were purchased from The Jackson Laboratory (Bar Harbor, ME, USA). Beginning one week later, these animals received a control or $5 \%$ BRB diet for 8 weeks: WT mice on the control diet $(n=5)$, KO mice on the control diet $(n=15)$, KO mice on $5 \%$ BRBs $(n=15)$. At the end of that period, colons were paraffin-embedded, and plasma and spleen were collected for the following assays.

\section{Isolation of murine splenocytes and detection of macrophages using flow cytometry}

Mice were euthanized by $\mathrm{CO}_{2}$ asphyxiation. Spleen was collected and placed into a wet $40 \mu \mathrm{m}$ cell strainer on top of a $50 \mathrm{~mL}$ centrifuge tube. Then the spleen was smashed in the cell strainer, which was rinsed with $5 \mathrm{~mL}$ of medium. The cell suspension collected in the tube was centrifuged at $1,000 \mathrm{rpm}$ for 5 minutes. The pellet was re-suspended in $5 \mathrm{~mL}$ red blood cell lysis buffer (containing $0.15 \mathrm{M} \mathrm{NH} 4 \mathrm{Cl}$, $0.01 \mathrm{M} \mathrm{KHCO}$, and $0.1 \mathrm{Mm}$ EDTA), and incubated at $4^{\circ} \mathrm{C}$ for 5 minutes. Then the cell suspension was centrifuged and rinsed with PBS (containing 5\% FBS). Splenocytes in the final pellet were suspended in RPMI1640 medium containing $20 \%$ FBS for further applications. A portion of the splenocytes was stained with surface-marker antibodies (BD Biosciences, 
Franklin Lakes, NJ, USA) for macrophages. The gating strategies were $C D 45^{+} \mathrm{CD} 11 \mathrm{~b}^{+} \mathrm{Gr}-1^{-} \mathrm{F} 4 / 80^{+}$. The samples were analyzed on an LSRII flow cytometer (BD Biosciences), and FlowJo (Tree Star, Ashland, OR, USA) was used to analyze the results. The data were presented as $\%$ positive cells.

\section{Real-time PCR}

mRNA was extracted from pathologically confirmed paraffin-embedded entire mouse colon tissues, using the RecoverAll $^{\mathrm{TM}}$ Total Nucleic Acid Isolation Kit for FFPE (Ambion, Grand Island, NY, USA). Two micrograms of total RNA per colon or splenocyte sample was reverse transcribed, using Superscript III RT (Invitrogen, Carlsbad, CA, USA). A TLR Superarray was purchased from Qiagen, Inc. (Venlo, Netherlands) to determine mRNA expression of 84 genes and the assay was performed according to the manufacturer's protocol. Mouse TLR-4, MYD-88, NF- $k B$, and COX-2 primers were purchased from IDT, Inc. (Coralville, IA, USA). These primers were validated and tested by IDT, Inc. and are commercially available for purchasing. The relative expression of a gene was determined by comparing the threshold cycle (Ct) of the gene against the $\mathrm{Ct}$ of the housekeeping gene glyceraldehyde 3-phosphate dehydrogenase (GAPDH).

\section{Human plasma}

Plasma samples from 10 healthy donors and 10 UC patients were obtained from the Cooperative Human Tissue Network (CHTN). Obtaining and using the specimens was approved by the Institutional Review Board of the Medical College of Wisconsin (PRO00016238).

\section{Measurement of eicosanoids and SAA in mouse and human plasma}

A portion of each mouse and human plasma sample was used to detect PGE2, PGI2, 15-LOX, 13-S-HODE, and SAA, using an enzyme immunoassay kit (MyBioSource, San Diego, CA, USA).

\section{Statistical analysis}

GraphPad Prism was used to analyze percentages of macrophages and mRNA levels of genes involved in TLR-4 signaling and levels of SAA, PGE2, PGI2, 15-LOX, and 13-S-HODE in plasma (unpaired, two-tailed $t$-test). A $P$-value less than 0.05 was considered statistically significant. Heatmap data
A

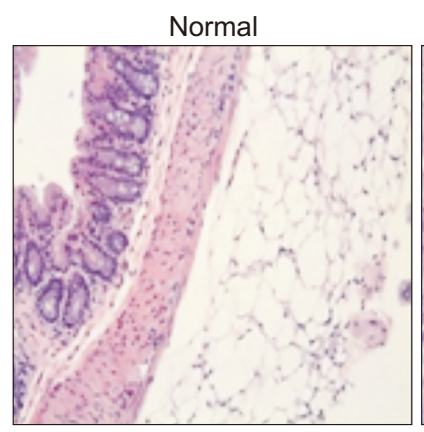

\section{Ulcerative colittis}

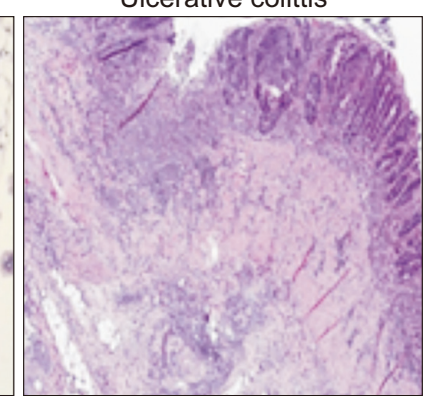

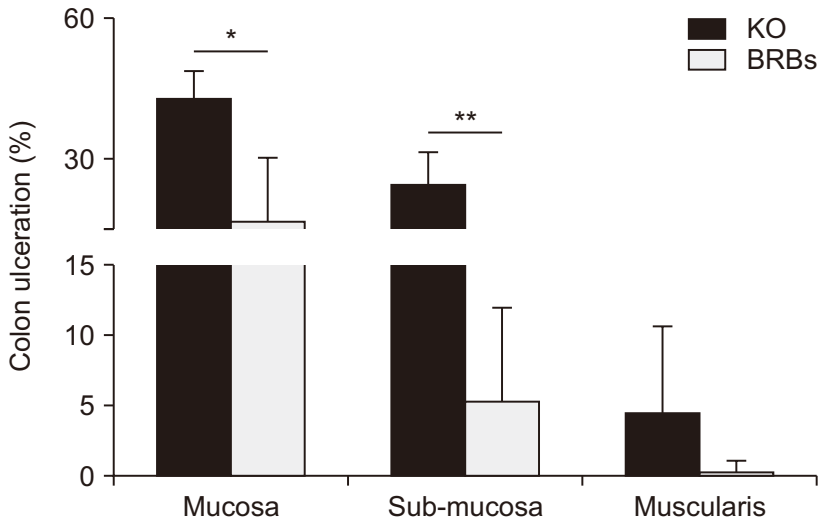

B

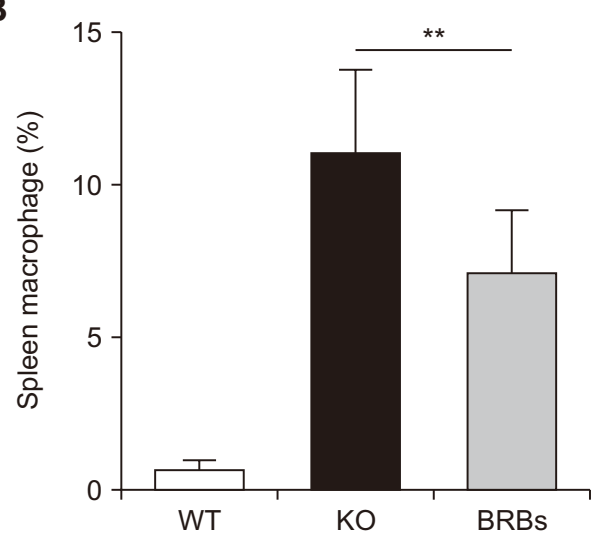

C

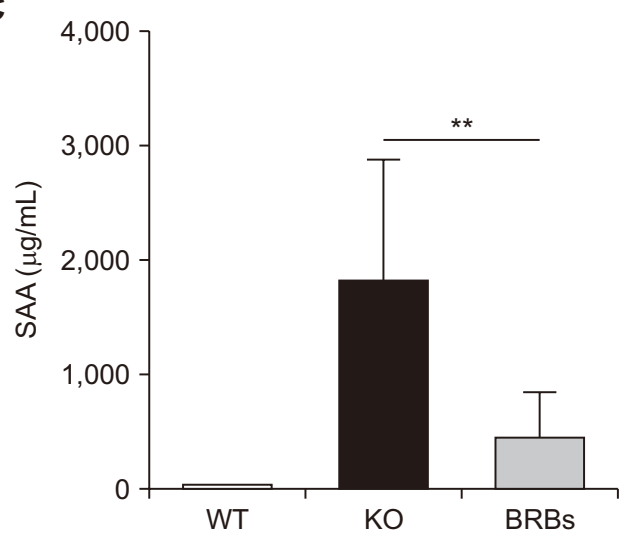

Figure 1. Black raspberries (BRBs) decreased colonic ulceration (A; H\&E, $\times 40$ ), the percentage of macrophages in spleen (B), and serum amyloid A (SAA) levels in plasma (C) of interleukin (IL)-10 knockout (KO) mice. Representative photos from colons of a wild type (WT) mouse and an IL-10 KO mouse bearing colitis were shown. WT, WT mice fed control diet; KO, IL-10 KO mice fed control diet; BRBs, IL-10 KO mice fed 5\% BRB diet. ${ }^{*} P<0.05,{ }^{* *} P<0.01$. 
were calculated as $(\mathrm{BRB} / \mathrm{WT}-\mathrm{KO} / \mathrm{WT}) /(\mathrm{KO} / \mathrm{WT}) \times 100$ and presented as percentages.

\section{RESULTS AND DISCUSSION}

\section{Dietary BRBs reduce colonic inflammation and decrease macrophage levels in spleen of IL-10 KO mice}

Representative H\&E staining of normal and UC colon tissues are shown in Figure 1A. Dietary administration of BRBs significantly reduced colon ulceration in mucosa and sub-mucosa (Fig. 1A). Ulceration was not observed in the colons of WT mice (data not shown). This result agrees with our previous study [3]. Whole spleen cells were analyzed for macrophage percentages, using flow-cytometry. The percentages of macrophages in the spleens of the IL-10 WT mice were much lower than in those of the KO mice, which were significantly reduced by BRBs (Fig. 1B). These results are important because bowel inflammation in IL-10 KO mice originates from uncontrolled immune responses stimulated by microbial antigens, and IL-10 is an essential immune regulator in the intestinal tract [9]. Furthermore, depletion of macrophages in IL-10 KO mice prevents the development of colitis $[10,11]$.
Our results suggest that BRBs' anti-inflammatory effects in IL$10 \mathrm{KO}$ mice associate with the ability to reduce macrophage levels. SAA, an inflammation marker, was measured in blood samples collected from both the WT and KO mice. As shown in Figure 1C, SAA levels were very low in the WT mice, and BRBs significantly decreased SAA in the KO mice. These results suggest that blood markers might be used to monitor response to $\mathrm{BRB}$ treatment.

\section{A BRB diet protectively modulates TLR-4 signaling in colon and spleen cells from IL-10 KO mice}

BRB treatment of IL-10 WT and IL-10 KO mice is described above. Whole cells of colons and spleens of 8-week-old WT and $\mathrm{KO}$ mice were collected, and mRNA was extracted from a portion of those cells for TLR Superarray (Qiagen, Inc.). A heatmap suggested that the BRB diet had differentially altered the expression of genes in TLR pathways (Fig. 2A). In general, the IL-10 KO mice had higher mRNA expression of TLR-4 and its downstream genes, MYD-88, NF- $\mathrm{KB}$, and COX-2, than the WT mice (Fig. 2B), and TLR-4 signaling was upregulated in their colon and spleens. This was likely stimulated and activated by microorganisms and their prod-
A

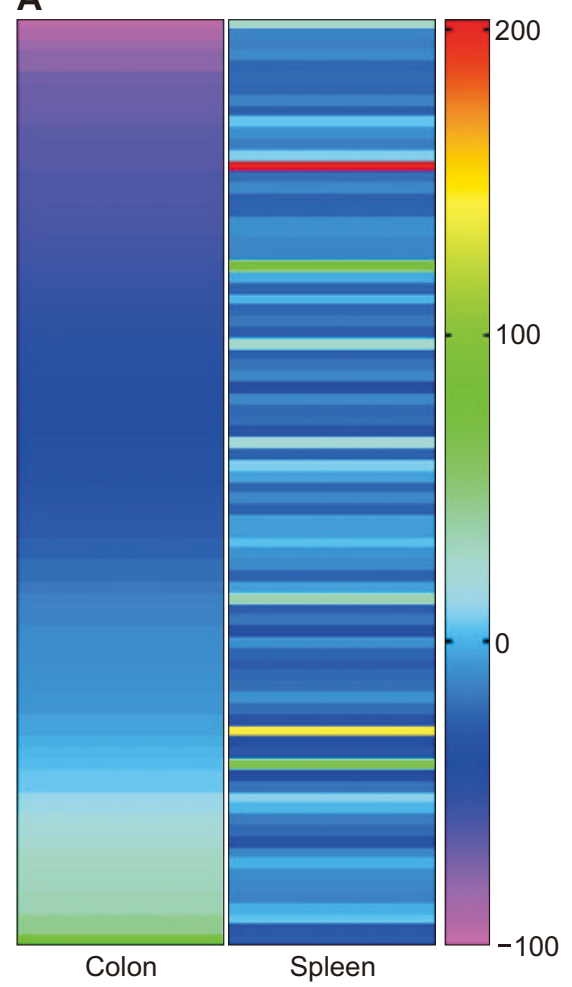

B
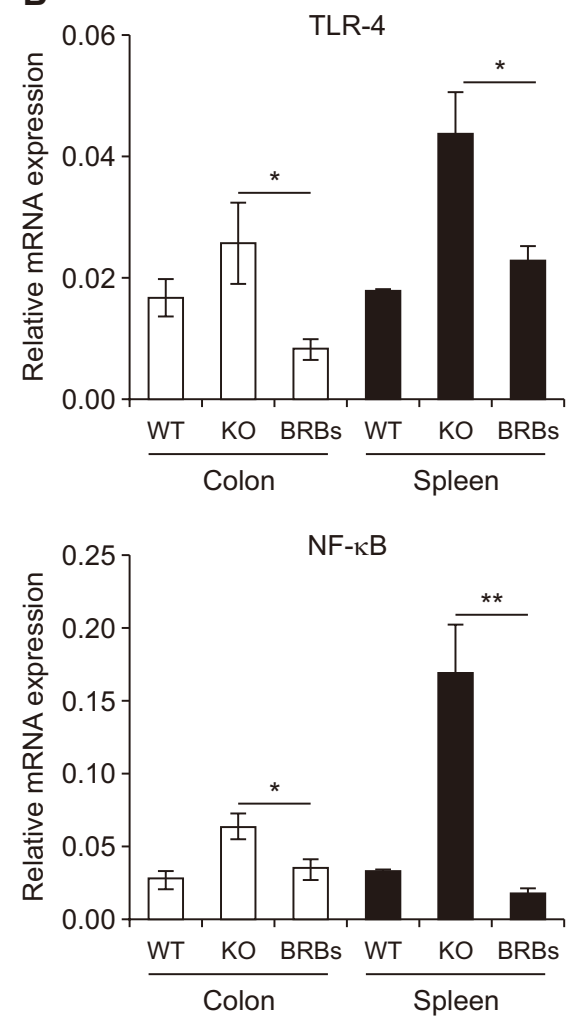

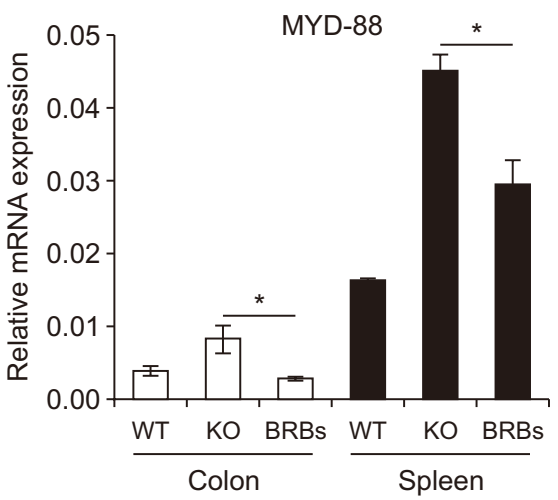

COX-2

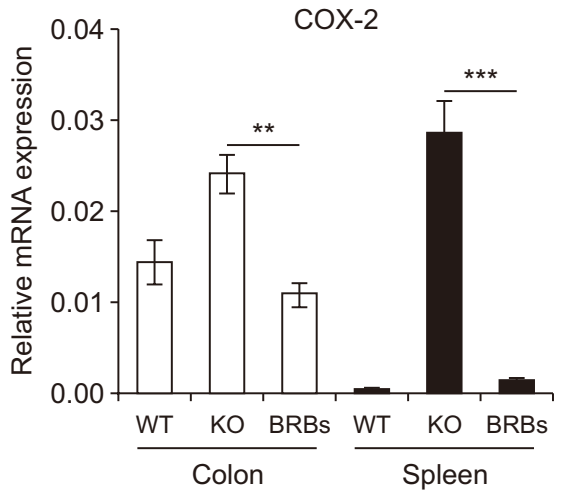

Figure 2. Black raspberries (BRBs) corrected dysregulated toll-like receptor (TLR)-4 signaling in colon and spleen of interleukin (IL)-10 knockout (KO) mice. Heatmap of BRB-induced changes in genes in TLR pathways (A) and mRNA expressions of TLR-4, MYD-88, NF- $\mathrm{kB}$, and COX-2 (B). WT, wild type (WT) mice fed control diet; KO, IL-10 KO mice fed control diet; BRBs, IL-10 KO mice fed $5 \%$ BRB diet. ${ }^{*} P<0.05,{ }^{* *} P<0.01$, ${ }^{* * *} P<0.001$. 
ucts, because UC has been suggested as a disease of gut dysbiosis [19]. However, BRBs significantly reduced mRNA expression of TLR-4 and its downstream genes in colons and spleens of the KO mice. Studies have shown that TLRs are broadly distributed in immune cells and intestinal epithelial cells, where they are the immune sensors of invading pathogens and microbial products [14]. They activate signaling pathways that induce the expression of immune and pro-inflammatory genes.

It is highly likely that BRBs regulate microorganisms and their products in the gut of IL-10 KO mice. Indeed, we have several lines of evidence that feeding BRBs alters the composition of the gut microbiota in WT rats [20] and increases benzoates that are produced when gut bacteria metabolize BRB components in WT mice [21], ApcMin ${ }^{-1}$ mice [22], and colorectal cancer patients [7]. Whether the presence of gut microorganisms is required for dietary BRBs to suppress colonic inflammation warrants further investigation.

\section{A BRB diet protectively modulates eicosanoids in circulating blood of IL-10 KO mice}

Data in Figure 2 show that dietary BRBs significantly de- creased COX-2 expression in the colon and spleen of IL$10 \mathrm{KO}$ mice. We then asked if BRBs could also regulate eicosanoids. Figure $3 \mathrm{~A}$ and $3 \mathrm{~B}$ shows that $\mathrm{BRB}$ s decreased PGE2 and PGI2 levels in the plasma of IL-10 KO mice. Previously, we showed that BRBs also decreased COX-2 mRNA expression in the esophagus and PGE2 levels in the plasma of carcinogen-treated rats [23]. Prostaglandins (e.g., PGI2) have been shown to affect macrophage recruitment [24], and UC patients have increased PGI2 protein expression [25]. Because BRBs decreased PGI2 (measured as 6 keto-PGF1alpha) in the plasma of IL-10 KO mice (Fig. 3B), it is likely that regulating $\mathrm{PGI} 2$ is one mechanism by which $\mathrm{BRBs}$ decrease the macrophage population in the spleen of IL-10 KO mice. Thus, it is clear that BRBs exert their anti-inflammatory effects by regulating eicosanoids in the COX pathway.

We then asked if BRBs could also regulate eicosanoids in the lipoxygenase pathway. Results showed that BRBs increased 15-LOX and its product, 13-S-HODE, in plasma of IL-10 KO mice (Fig. 3C and 3D). Therefore, BRBs protectively regulated both the COX and lipoxygenase pathways. In comparison with healthy individuals, 15-LOX and 13-S-HODE levels were decreased in plasma of UC patients (Fig. 4) sug-
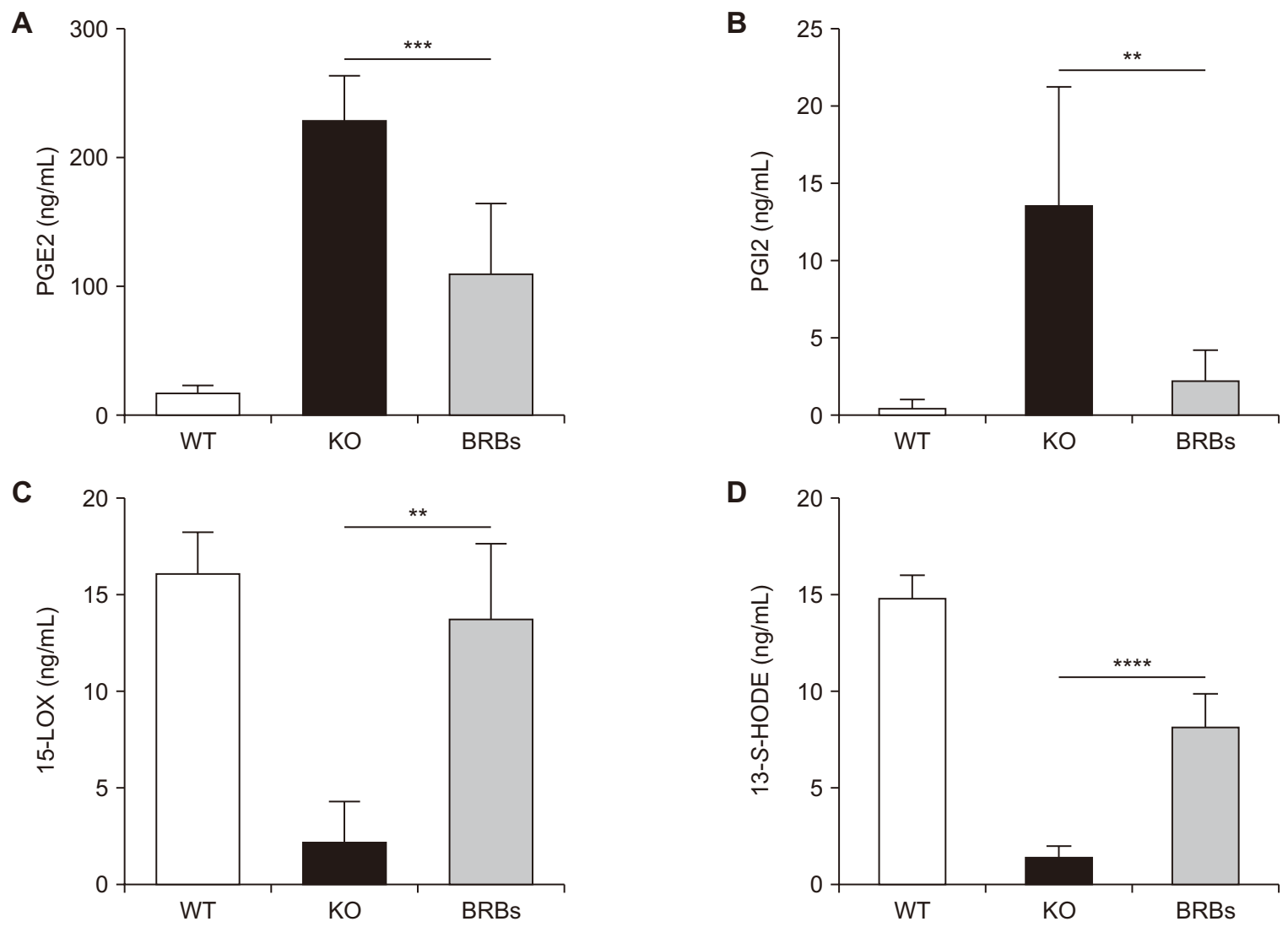

Figure 3. Black raspberries (BRBs) decreased prostaglandin E2 (PGE2) (A) and prostaglandin I2 (PGI2) (B) levels and increased 15-lipoxygenase (15-LOX) (C), and 13-S-hydroxyoctadecadienoic acid (13-S-HODE) (D) levels in plasma of interleukin (IL)-10 knockout (KO) mice. WT, wild type (WT) mice fed control diet; KO, IL-10 KO mice fed control diet; BRBs, IL-10 KO mice fed $5 \%$ BRB diet. ${ }^{* *} P<0.01,{ }^{* * *} P<0.001$, ${ }^{* * \star *} P<0.0001$. 
Huang et al.
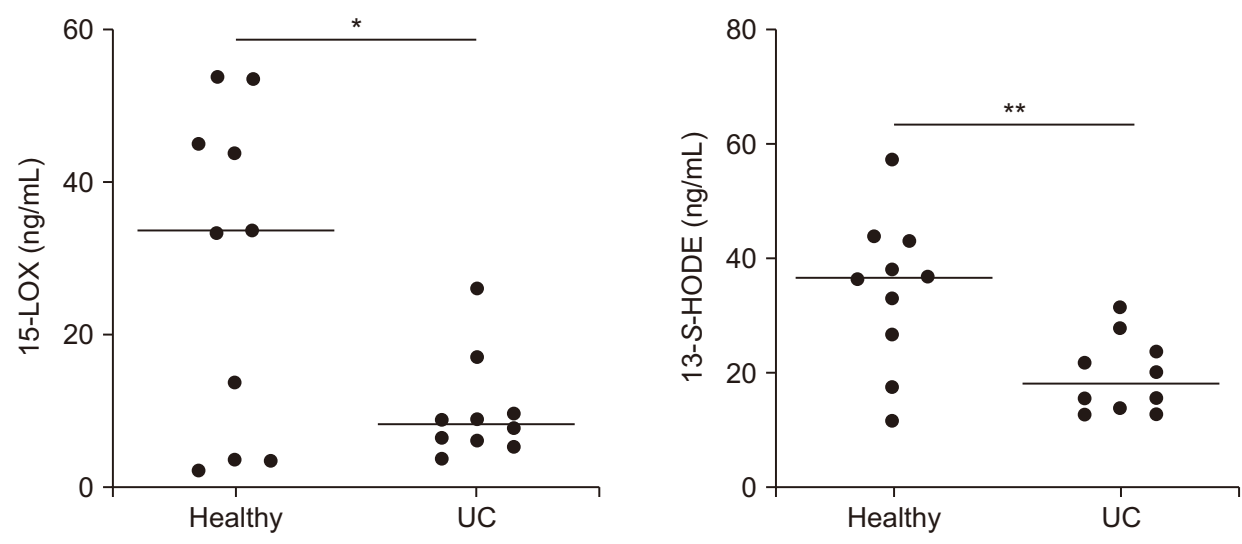

Figure 4. Levels of 15-lipoxygenase (15-LOX) and 13-S-hydroxyoctadecadienoic acid (13-S-HODE) in plasma from 10 healthy individuals and 10 ulcerative colitis (UC) patients. ${ }^{*} P<0.05,{ }^{* *} P<0.01$.

gesting a dysregulated lipoxygenase pathway. Therefore, these markers could be used in future trials of BRB intervention in UC patients.

Studies have suggested that marine fish-oil supplements, rich in n-3 fatty acids, may decrease colonic inflammation in UC by increasing the synthesis of anti-inflammatory eicosanoids [26]. Indeed, we showed that the BRB diet contained 30-fold higher levels of linolenate than the control diet, resulting in significantly increased levels of multiple n-3 polyunsaturated fatty acids, including stearidonate, docosapentaenoate, eicosapentaenoate, and docosahexaenoate, in livers of BRBfed mice [21]. Therefore, our results suggest that BRBs regulate both the COX and lipoxygenase pathways to suppress colonic inflammation in UC.

\section{CONCLUSIONS}

UC is a chronic inflammatory disease of the colon with a steadily rising prevalence in Western and newly industrialized countries [1]. A meta-analysis of 14 case-control studies suggested that higher consumption of fruits and vegetables associated inversely with the risk of UC [27]. Although the importance of such a diet to the etiology of UC is beginning to be appreciated [27], the protective mechanisms remain largely unclear. Using berries as an example, we showed that BRBs decreased the percentage of macrophages in spleens of IL-10 KO mice. Mechanistically, we showed that a BRB diet corrected dysregulated TLR4 signaling in cells from the colon and spleen, decreased PGE2 and PGI2, and increased 15-LOX and its product, 13-S-HODE, in plasma of IL-10 KO mice. This anti-inflammatory mechanism for BRBs that we have uncovered could well apply to many other commonly consumed fruits and vegetables.

\section{ACKNOWLEDGMENTS}

This work was supported by NIH grants CA148818 and USDA/NIFA 2020-67017-30843 (to L.-S. Wang), and
CA185301, Al129582 and NS106170 (to J. Yu).

\section{CONFLICTS OF INTEREST}

No potential conflicts of interest were disclosed.

\section{ORCID}

Yi-Wen Huang, https://orcid.org/0000-0001-9220-8770

Carla Elena Echeveste, https://orcid.org/0000-0001-8442-0683

Kiyoko Oshima, https://orcid.org/0000-0002-3357-6247

Jianying Zhang, https://orcid.org/0000-0001-5896-9509

Martha Yearsley, https://orcid.org/0000-0003-0352-1277

Jianhua Yu, https://orcid.org/0000-0002-0326-3223

Li-Shu Wang, https://orcid.org/0000-0002-6500-6943

\section{REFERENCES}

1. Kaplan GG, Ng SC. Understanding and preventing the global increase of inflammatory bowel disease. Gastroenterology 2017;152:313-21.e2.

2. $\mathrm{Ng} \mathrm{SC}, \mathrm{Kamm}$ MA. Therapeutic strategies for the management of ulcerative colitis. Inflamm Bowel Dis 2009;15:935-50.

3. Wang LS, Kuo CT, Huang TH, Yearsley M, Oshima K, Stoner $\mathrm{GD}$, et al. Black raspberries protectively regulate methylation of Wnt pathway genes in precancerous colon tissue. Cancer Prev Res (Phila) 2013;6:1317-27.

4. Wang LS, Kuo CT, Stoner K, Yearsley M, Oshima K, Yu J, et al. Dietary black raspberries modulate DNA methylation in dextran sodium sulfate (DSS)-induced ulcerative colitis. Carcinogenesis 2013;34:2842-50.

5. Montrose DC, Horelik NA, Madigan JP, Stoner GD, Wang LS, Bruno RS, et al. Anti-inflammatory effects of freeze-dried black raspberry powder in ulcerative colitis. Carcinogenesis 2011;32:343-50.

6. Wang LS, Arnold M, Huang YW, Sardo C, Seguin C, Martin E, et al. Modulation of genetic and epigenetic biomarkers of colorectal 
cancer in humans by black raspberries: a phase I pilot study. Clin Cancer Res 2011;17:598-610.

7. Pan P, Skaer CW, Stirdivant SM, Young MR, Stoner GD, Lechner JF, et al. Beneficial regulation of metabolic profiles by black raspberries in human colorectal cancer patients. Cancer Prev Res (Phila) 2015;8:743-50.

8. Wang LS, Burke CA, Hasson H, Kuo CT, Molmenti CL, Seguin $\mathrm{C}$, et al. A phase Ib study of the effects of black raspberries on rectal polyps in patients with familial adenomatous polyposis. Cancer Prev Res (Phila) 2014;7:666-74.

9. Kühn R, Löhler J, Rennick D, Rajewsky K, Müller W. Interleukin10-deficient mice develop chronic enterocolitis. Cell 1993;75:26374.

10. Kamada N, Hisamatsu T, Okamoto S, Sato T, Matsuoka K, Arai $\mathrm{K}$, et al. Abnormally differentiated subsets of intestinal macrophage play a key role in Th1-dominant chronic colitis through excess production of IL-12 and IL-23 in response to bacteria. J Immunol 2005;175:6900-8.

11. Sheikh SZ, Plevy SE. The role of the macrophage in sentinel responses in intestinal immunity. Curr Opin Gastroenterol 2010;26:578-82.

12. Schreiber S, Fedorak RN, Wild G, Gangl A, Targan S, Jacyna $M$, et al. Safety and tolerance of rHulL-10 treatment in patients with mild/moderate active ulcerative colitis. Gastroenterology 1998;114 Suppl 1:A1080-1.

13. Moore KW, de Waal Malefyt R, Coffman RL, O'Garra A. Interleukin-10 and the interleukin-10 receptor. Annu Rev Immunol 2001;19:683-765.

14. Abreu MT. Toll-like receptor signalling in the intestinal epithelium: how bacterial recognition shapes intestinal function. Nat Rev Immunol 2010;10:131-44.

15. Liew FY, Xu D, Brint EK, O'Neill LA. Negative regulation of Tolllike receptor-mediated immune responses. Nat Rev Immunol 2005;5:446-58.

16. Szumilas D, Krysiak R, Okopień B. [The role of TLR4 receptor in development of inflammation and carcinogenesis in ulcerative colitis and pharmacotherapy of this disorder]. Wiad Lek 2013;66:3-9. Polish.
17. Biswas A, Wilmanski J, Forsman H, Hrncir T, Hao L, TlaskalovaHogenova $\mathrm{H}$, et al. Negative regulation of Toll-like receptor signaling plays an essential role in homeostasis of the intestine. Eur J Immunol 2011;41:182-94.

18. Swirski FK, Nahrendorf M, Etzrodt M, Wildgruber M, CortezRetamozo V, Panizzi P, et al. Identification of splenic reservoir monocytes and their deployment to inflammatory sites. Science 2009;325:612-6.

19. Shen ZH, Zhu CX, Quan YS, Yang ZY, Wu S, Luo WW, et al. Relationship between intestinal microbiota and ulcerative colitis: mechanisms and clinical application of probiotics and fecal microbiota transplantation. World J Gastroenterol 2018;24:5-14.

20. Pan P, Lam V, Salzman N, Huang YW, Yu J, Zhang J, et al. Black raspberries and their anthocyanin and fiber fractions alter the composition and diversity of gut microbiota in F-344 rats. Nutr Cancer 2017;69:943-51.

21. Pan P, Skaer CW, Wang HT, Kreiser MA, Stirdivant SM, Oshima $\mathrm{K}$, et al. Systemic metabolite changes in wild-type C57BL/6 mice fed black raspberries. Nutr Cancer 2017;69:299-306.

22. Pan P, Skaer CW, Wang HT, Stirdivant SM, Young MR, Oshima $\mathrm{K}$, et al. Black raspberries suppress colonic adenoma development in ApcMin/+ mice: relation to metabolite profiles. Carcinogenesis 2015;36:1245-53.

23. Wang LS, Hecht SS, Carmella SG, Yu N, Larue B, Henry C, et al. Anthocyanins in black raspberries prevent esophageal tumors in rats. Cancer Prev Res (Phila) 2009;2:84-93.

24. Kuo CH, Ko YC, Yang SN, Chu YT, Wang WL, Huang SK, et al. Effects of PGI2 analogues on Th1- and Th2-related chemokines in monocytes via epigenetic regulation. $\mathrm{J} \mathrm{Mol} \mathrm{Med} \mathrm{(Berl)}$ 2011;89:29-41.

25. McCartney SA, Mitchell JA, Fairclough PD, Farthing MJ, Warner TD. Selective COX-2 inhibitors and human inflammatory bowel disease. Aliment Pharmacol Ther 1999;13:1115-7.

26. Ross $\mathrm{E}$. The role of marine fish oils in the treatment of ulcerative colitis. Nutr Rev 1993;51:47-9.

27. Li F, Liu X, Wang W, Zhang D. Consumption of vegetables and fruit and the risk of inflammatory bowel disease: a meta-analysis. Eur J Gastroenterol Hepatol 2015;27:623-30. 